W. F. Pfeffer, Department of Mathematics, University of California, Davis, CA 95616. e-mail: wfpfeffer@ucdavis.edu

\title{
THE STOKES THEOREM FOR THE GENERALIZED RIEMANN INTEGRAL
}

\begin{abstract}
In $\mathbb{R}^{m}$, we define the generalized Riemann integral over normal $m$ dimensional currents with compact support and bounded multiplicities, and prove the Stokes theorem for continuous $(m-1)$-forms that are pointwise Lipschitz outside sets of $\sigma$-finite $(m-1)$-dimensional Hausdorff measure. In addition, we show that the usual transformation formula holds for local lipeomorphisms, which need not be injective.
\end{abstract}

\section{Introduction}

Using the change of variables formula of [6, Theorem 6.7] and standard techniques [8, Chapter 5], it is easy to lift the generalized Riemann integral defined in [6, Definition 5.1] to a compact oriented Lipschitz manifold $M$ with boundary and establish the Stokes theorem for pointwise Lipschitz $(m-1)$-forms on $M$. In this note we prove that the same is true for certain top-dimensional currents. We also show that the generalized Riemann integral transforms well with respect to local lipeomorphisms, which need not be injective. It is our hope that eventually similar results may be obtained in middle dimensions.

\section{Preliminaries}

The set of all real numbers is denoted by $\mathbb{R}$, and the ambient space of this note is $\mathbb{R}^{m}$ where $m \geq 1$ is a fixed integer. The open ball of radius $r>0$ about $x \in \mathbb{R}^{m}$ is denoted by $B(x, r)$. The symbols cl $E, \partial E$, and $d(A)$ denote, respectively, the closure, boundary, and diameter of a set $E \subset \mathbb{R}^{m}$.

Lebesgue measure in $\mathbb{R}^{m}$ is denoted by $\mathcal{L}^{m}$; however, for $E \subset \mathbb{R}^{m}$ we write $|E|$ instead of $\mathcal{L}^{m}(E)$. The essential closure $\mathrm{cl}_{*} E$ and essential boundary $\partial_{*} E$

Key Words: BV sets and functions, local lipeomorphisms, top-dimensional normal currents

Mathematical Reviews subject classification: Primary: 49Q15, 28A75. Secondary: 26B15

Received by the editors July 10, 2000 
of a measurable set $E \subset \mathbb{R}^{m}$ are the sets of all $x \in \mathbb{R}^{m}$ at which the density of $E$ lies in $(0,1]$ and $(0,1)$, respectively [4, Section 1.7.1]. A thin set is a set $S \subset \mathbb{R}^{m}$ whose $(m-1)$-dimensional Hausdorff measure $\mathcal{H}^{m-1}$ is $\sigma$-finite.

Unless specified otherwise, all functions we consider are real-valued. By $\chi_{E}$ we denote the indicator of a set $E \subset \mathbb{R}^{m}$. If $E \subset \mathbb{R}^{m}$ is a measurable set and $f \in L^{1}(E)$, we denote by $(L) \int_{E} f d \mathcal{L}^{m}$ or $(L) \int_{E} f(x) d x$ the Lebesgue integral of $f$ over $E$.

The linear space of all locally bounded locally BV functions in $\mathbb{R}^{m}$ is denoted by $B V_{\text {loc }}^{\infty}\left(\mathbb{R}^{m}\right)$, and the linear space of all bounded BV functions in $\mathbb{R}^{m}$ with compact support is denoted by $B V_{c}^{\infty}\left(\mathbb{R}^{m}\right)$. If $g \in B V_{c}^{\infty}\left(\mathbb{R}^{m}\right)$, we denote by $|g|_{1},|g|_{\infty}, D g$, and $\|g\|$ the $L^{1}$ norm, $L^{\infty}$ norm, distributional gradient, and total variation of $g$, respectively. If $g \in B V_{\mathrm{loc}}^{\infty}\left(\mathbb{R}^{m}\right)$ and $v: \mathbb{R}^{m} \rightarrow \mathbb{R}^{m}$ is a $C^{1}$ vector field with compact support, then

$$
\int_{\mathbb{R}^{m}} g \operatorname{div} v d \mathcal{L}^{m}=-\int_{\mathbb{R}^{m}} v \cdot d(D g) .
$$

For $n=1,2, \ldots$, the $L^{1}$ norm $|\cdot|_{1}$ gives the family

$$
B V_{n}:=\left\{g \in B V_{c}^{\infty}\left(\mathbb{R}^{m}\right): \operatorname{supp} g \subset \operatorname{cl} B(0, n) \text { and }\|g\|+|g|_{\infty} \leq n+1\right\}
$$

a compact topology. The largest topology $\tau$ in $B V_{c}^{\infty}\left(\mathbb{R}^{m}\right)$ for which all inclusion maps $B V_{n} \hookrightarrow B V_{c}^{\infty}$ are continuous is a nonmetrizable locally convex Hausdorff topology that is sequential and sequentially complete. Given a locally $\mathrm{BV}$ set $E \subset \mathbb{R}^{m}$, we denote by $\mathcal{B V}(E)$ the family of all bounded BV subsets of $E$. Identifying each $A \in \mathcal{B} \mathcal{V}(E)$ with its indicator $\chi_{A} \in B V_{c}^{\infty}\left(\mathbb{R}^{m}\right)$, we view $\mathcal{B} \mathcal{V}(E)$ as a closed subspace of $\left(B V_{c}^{\infty}\left(\mathbb{R}^{m}\right), \tau\right)$.

Remark 2.1. A clear self-contained exposition of BV functions and BV sets can be found in Chapter 5 of [4] or [9]. As usual, when we talk about spaces of BV functions or BV sets, their elements are the equivalence classes of BV functions or BV sets, respectively. On the other hand, an individual BV function or BV set is normally not identified with its equivalence class.

The unit exterior normal of a BV set $A$ is denoted by $\nu_{A}$. The Gauss-Green formula

$$
\int_{A} \operatorname{div} v d \mathcal{L}^{m}=\int_{\partial_{*} A} v \cdot \nu_{A} d \mathcal{H}^{m-1}
$$

holds for each $C^{1}$ vector field $v: \mathbb{R}^{m} \rightarrow \mathbb{R}^{m}$ with compact support. The perimeter of a bounded $\mathrm{BV}$ set $A$ is denoted by $\|A\|$, and the number

$$
r(A):= \begin{cases}\frac{|A|}{d(A)\|A\|} & \text { if } d(A)\|A\|>0 \\ 0 & \text { otherwise }\end{cases}
$$

is called the regularity of $A$. 


\section{The R-Integral}

A charge is an additive $\tau$-continuous function defined on $\mathcal{B} \mathcal{V}\left(\mathbb{R}^{m}\right)$. Explicitly, a charge is an additive function $F$ on $\mathcal{B} \mathcal{V}\left(\mathbb{R}^{m}\right)$ having the following property: given $\varepsilon>0$, there is a $\delta>0$ such that $|F(A)|<\varepsilon$ for each BV set $A \subset$ $B(0,1 / \varepsilon)$ with $\|A\|<1 / \varepsilon$ and $|A|<\delta$. If $F$ is a charge and $E \subset \mathbb{R}^{m}$ is a locally $\mathrm{BV}$ set, the function $F \mathrm{~L} E$ defined by

$$
(F\llcorner E)(A):=F(A \cap E)
$$

for each $A \in \mathcal{B} \mathcal{V}\left(\mathbb{R}^{m}\right)$ is also a charge. We say $F$ is a charge in $E$ whenever $F=F \mathrm{~L} E$. Typical examples of a charge are an absolutely continuous signed measure and the flux of a continuous vector field.

A nonnegative function $\delta$ defined on a set $E \subset \mathbb{R}^{m}$ is called a gage on $E$ whenever $\{\delta=0\}$ is a thin set. A partition is a collection (possibly empty)

$$
P:=\left\{\left(A_{1}, x_{1}\right), \ldots,\left(A_{p}, x_{p}\right)\right\}
$$

where $A_{1}, \ldots, A_{p}$ are disjoint bounded BV sets, and $x_{1}, \ldots, x_{p}$ are points of $\mathbb{R}^{m}$. Given $\varepsilon>0$ and a gage $\delta$ on $E \subset \mathbb{R}^{m}$, the partition $P$ is called

- $\varepsilon$-regular if $r\left(A_{i} \cup\left\{x_{i}\right\}\right)>\varepsilon$ for $i=1, \ldots, p$,

- $\delta$-fine if $x_{i} \in E$ and $d\left(A_{i} \cup\left\{x_{i}\right\}\right)<\delta\left(x_{i}\right)$ for $i=1, \ldots, p$.

Definition 3.1. Let $E$ be a locally BV set. A function $f$ defined on $\mathrm{cl}_{*} E$ is called $R$-integrable in $E$ whenever there is a charge $F$ in $E$ having the following property: given $\varepsilon>0$, we can find a gage $\delta$ on $B(0,1 / \varepsilon) \cap \mathrm{cl}_{*} E$ so that

$$
\sum_{i=1}^{p}\left|f\left(x_{i}\right)\right| A_{i}\left|-F\left(A_{i}\right)\right|<\varepsilon
$$

for each $\varepsilon$-regular $\delta$-fine partition $\left\{\left(A_{1}, x_{1}\right), \ldots,\left(A_{p}, x_{p}\right)\right\}$.

The charge $F$ from Definition 3.1 is called the $R$-primitive of $f$. For a bounded BV set $A \subset E$, we denote $F(A)$ by the symbol $(R) \int_{A} f d \mathcal{L}^{m}$, or $(R) \int_{A} f(x) d x$, and call this number the R-integral of $f$ over $A$. According to [6, Corollary 5.9], the R-integrability of $f$ and the R-primitive of $f$ depend only on the values $f$ takes almost everywhere in $E$. In particular, we can talk about the R-integrability, R-primitive, and R-integral of a function defined only almost everywhere in $E$. The linear space of all R-integrable functions in $E$ is denoted by $R(E)$. 
It follows from [6] that the R-integral has the usual properties such as linearity, monotonicity, etc., associated with the word "integral." If $f$ is a function defined on $E$ and $f \in L^{1}(A)$ for each bounded BV set $A \subset E$, then $f \in R(E)$ and the Lebesgue primitive of $f$

$$
F: A \mapsto(L) \int_{A \cap E} f d \mathcal{L}^{m}: \mathcal{B} \mathcal{V}\left(\mathbb{R}^{m}\right) \rightarrow \mathbb{R}
$$

coincides with the R-primitive of $f$. For this reason we often denote by $\int$ both symbols $(L) \int$ and $(R) \int$.

We say a charge $F$ is derivable at $x \in \mathbb{R}^{m}$ if a finite $\lim \left[F\left(B_{n}\right) /\left|B_{n}\right|\right]$ exists for each sequence $\left\{B_{n}\right\}$ in $\mathcal{B} \mathcal{V}$ such that

$$
\lim d\left(B_{n} \cup\{x\}\right)=0 \text { and } \inf r\left(B_{n} \cup\{x\}\right)>0 .
$$

If all these limits exist, they have the same value called the derivate of $F$ at the point $x$, denoted by $F^{\prime}(x)$.

For a charge $F$ and a set $E \subset \mathbb{R}^{m}$, let

$$
V_{*} F(E):=\sup _{\varepsilon>0} \inf _{\delta} \sup _{P} \sum_{i=1}^{p}\left|F\left(A_{i}\right)\right|
$$

where $\delta$ is a gage on $E$, and $P=\left\{\left(A_{1}, x_{1}\right), \ldots,\left(A_{p}, x_{p}\right)\right\}$ is an $\varepsilon$-regular $\delta$-fine partition. It was shown in [1] that the extended real-valued function

$$
V_{*} F: E \mapsto V_{*} F(E)
$$

is a Borel regular measure in $\mathbb{R}^{m}$ that coincides with the classical variation of $F$ on each compact interval.

A charge $F$ in a locally $\mathrm{BV}$ set $E$ is called, respectively, $\mathrm{AC}_{*}$ or $\mathrm{AC}$ if the measure $\left(V_{*} F\right) \mathrm{L} \mathrm{cl}_{*} E$ is absolutely continuous or absolutely continuous and locally finite. According to [2], each $\mathrm{AC}_{*}$ charge, and a fortiori each $\mathrm{AC}$ charge, in $E$ is derivable almost everywhere in $E$. Moreover, the following theorem holds.

Theorem 3.2. If $F$ is a charge in a locally $B V$ set $E$, then

(i) $F$ is $A C$ if and only if $F$ is the Lebesgue primitive of $F^{\prime}$;

(ii) $F$ is $A C_{*}$ if and only if $F$ is the $R$-primitive of $F^{\prime}$.

Let $E \subset \mathbb{R}^{m}$ be a measurable set. We say a vector field $v: E \rightarrow \mathbb{R}^{m}$ is differentiable relative to $E$ at $x \in \operatorname{int}_{*} E$ if there is a linear map $D_{E} v(x)$ : $\mathbb{R}^{m} \rightarrow \mathbb{R}^{m}$ satisfying the following condition: given $\varepsilon>0$, there is a $\delta>0$ such that

$$
\left|v(y)-v(x)-D_{E} v(x)(y-x)\right|<\varepsilon|y-x|
$$

for each $y \in E \cap B(x, \delta)$. Suppose $v$ is differentiable relative to $E$ at $x \in$ 
$\operatorname{int}_{*} E$. Then $v$ is approximately differentiable at $x$ and $D_{E} v(x)=$ ap $D v(x)$; in particular, $D_{E} v(x)$ is unique [5, Section 3.1]. The trace of $D_{E} v(x)$ is called the divergence of $v$ at $x$ relative to $E$, still denoted by $\operatorname{div} v(x)$.

If $C \subset \mathbb{R}^{m}$ is a closed set, and if $v: C \rightarrow \mathbb{R}^{m}$ is a continuous vector field that is Lipschitz at each point of a set $S \subset \operatorname{int}_{*} C$, then using a weak version of Whitney's extension theorem [7, Proposition 13.4.4] and Stepanoff's theorem [5, Theorem 3.1.9], it is easy to see that $v$ is differentiable relative to $C$ at almost all points of $S$.

The following version of the Gauss-Green theorem has been established in [6, Theorem 5.19].

Theorem 3.3. Let $A$ be a bounded $B V$ set, and let $v: \operatorname{cl} A \rightarrow \mathbb{R}^{m}$ be a continuous vector field. If $v$ is Lipschitz at each $x \in A-S$ where $S$ is a thin set, then $\operatorname{div} v$ belongs to $R(A)$ and

$$
(R) \int_{A} \operatorname{div} v d \mathcal{L}^{m}=(L) \int_{\partial_{*} A} v \cdot \nu d \mathcal{H}^{m-1} .
$$

\section{The Stokes Theorem}

We shall need a result established in [3, Proposition 3.5].

Proposition 4.1. Let $F$ be a charge in a locally $B V$ set $E \subset \mathbb{R}^{m}$, and let $A$ be a set in $\mathcal{B} \mathcal{V}\left(\mathbb{R}^{m+1}\right)$. For $\mathcal{L}^{1}$-almost all $t \in \mathbb{R}$, the section

$$
A^{t}:=\left\{x \in \mathbb{R}^{m}:(x, t) \in A\right\}
$$

belongs to $\mathcal{B V}\left(\mathbb{R}^{m}\right)$, and the function $t \mapsto F\left(A^{t}\right)$ belongs to $L^{1}(\mathbb{R})$. Letting

$$
\left(F \times \mathcal{L}^{1}\right)(A):=\int_{\mathbb{R}} F\left(A^{t}\right) d t
$$

for each $A \in \mathcal{B V} \mathcal{V}\left(\mathbb{R}^{m+1}\right)$ defines a charge $F \times \mathcal{L}^{1}$ in $E \times \mathbb{R}$.

For a nonnegative function $g \in B V_{c}^{\infty}\left(\mathbb{R}^{m}\right)$, the set

$$
\Sigma_{g}:=\left\{(x, t) \in \mathbb{R}^{m} \times \mathbb{R}: 0<t<g(x)\right\}
$$

belongs to $\mathcal{B V}\left(\mathbb{R}^{m+1}\right)$, and $\left(\Sigma_{g}\right)^{t}=\{g>t\}$ for every $t>0$. We let

$$
\begin{aligned}
\langle F, g\rangle & :=\left(F \times \mathcal{L}^{1}\right)\left(\Sigma_{g^{+}}\right)-\left(F \times \mathcal{L}^{1}\right)\left(\Sigma_{g^{-}}\right) \\
& =\int_{0}^{\infty} F(\{g>t\}) d t-\int_{0}^{\infty} F(\{-g>t\}) d t
\end{aligned}
$$

for each charge $F$ and each $g \in B V_{c}^{\infty}\left(\mathbb{R}^{m}\right)$. For the proof of the next proposition we refer to [3, Proposition 4.1]. 
Proposition 4.2. For each charge $F$, the map $\Theta(F): g \mapsto\langle F, g\rangle$ is a $\tau$ continuous linear functional on $B V_{c}^{\infty}\left(\mathbb{R}^{m}\right)$. The map $F \mapsto \Theta(F)$ is a linear bijection from the space of all charges onto the dual space of $\left(B V_{c}^{\infty}\left(\mathbb{R}^{m}\right), \tau\right)$.

If $g \in B V_{\text {loc }}^{\infty}\left(\mathbb{R}^{m}\right)$, then $g \chi_{A} \in B V_{c}^{\infty}\left(\mathbb{R}^{m}\right)$ for each $A \in \mathcal{B V}\left(\mathbb{R}^{m}\right)$. Thus given a charge $F$ and a $g \in B V_{\text {loc }}^{\infty}\left(\mathbb{R}^{m}\right)$, we can define a charge

$$
F\left\llcorner g: A \mapsto\left\langle F, g \chi_{A}\right\rangle: \mathcal{B V}\left(\mathbb{R}^{m}\right) \rightarrow \mathbb{R} .\right.
$$

It is easy to see that $(F \mathbf{L} g) \mathbf{L} h=F \mathbf{L}(g h)$ for each $h \in B V_{\text {loc }}^{\infty}\left(\mathbb{R}^{m}\right)$, and the following theorem follows from [3, Section 6].

Theorem 4.3. Let $E$ be a locally $B V$ set, and let $g \in B V_{\mathrm{loc}}^{\infty}\left(\mathbb{R}^{m}\right)$. If $F$ is an $A C_{*}$ charge in $E$, then so is $F \mathrm{~L} g$ and $(F \mathrm{~L} g)^{\prime}(x)=F^{\prime}(x) g(x)$ for almost all $x \in E$.

As usual we denote by $d x_{1}, \ldots, d x_{m}$ the dual base to the standard base of $\mathbb{R}^{m}$, and orient $\mathbb{R}^{m}$ by the volume element $d x:=d x_{1} \wedge \cdots \wedge d x_{m}$. The $m$-form $\theta:=f d x$ defined almost everywhere in a locally BV set $E$ is called R-integrable whenever $f \in R(E)$. Let

$$
\omega:=\sum_{i=1}^{m}(-1)^{i-1} w_{i} d x_{1} \wedge \cdots \widehat{d x_{i}} \cdots \wedge d x_{m}
$$

where $w_{\omega}=\left(w_{1} \ldots, w_{m}\right)$ is a vector field defined on a locally BV set $E$. We say $\omega$ is $C^{\infty}$, or continuous, or pointwise Lipschitz whenever $w_{\omega}$ is $C^{\infty}$, or continuous, or pointwise Lipschitz, respectively. If $w_{\omega}$ is differentiable relative to $E$ at an $x \in \operatorname{int}_{*} E$, we let

$$
d \omega(x):=\operatorname{div} w_{\omega}(x) d x .
$$

We shall use freely the notation of [5, Chapter 4]. For a set $E \subset \mathbb{R}^{m}$, we denote by $\mathbf{N}_{m}^{c, \infty}(E)$ the algebra of all $m$-dimensional currents $\mathbf{E}^{m} \mathbf{L} g$ associated with those $g \in B V_{c}^{\infty}\left(\mathbb{R}^{m}\right)$ for which $\{g \neq 0\} \subset E$. Identifying $\mathbf{E}^{m} \mathbf{L} g$ with $g$, we view $\mathbf{N}_{m}^{c, \infty}(E)$ as a closed subspace of $\left(B V_{c}^{\infty}\left(\mathbb{R}^{m}\right), \tau\right)$.

Let $E$ be a locally BV set, and let $T:=\mathbf{E}^{m} \mathbf{L} g$ belong to $\mathbf{N}_{m}^{c, \infty}(E)$. If $\theta:=f d x$ is an R-integrable $m$-form in $E$, we find a bounded BV set $A \subset E$ with $\{g \neq 0\} \subset A$ and let

$$
\int_{T} \theta:=\int_{A} f g d \mathcal{L}^{m}
$$

Note that $f g \in R(A)$ by Theorem 4.3, and that the definition of $\int_{T} \theta$ does not depend on the choice of $A$. Moreover, $\int_{T} \theta=\langle T, \theta\rangle$ for each $C^{\infty} m$-form $\theta$ in $\mathbb{R}^{m}$ with compact support. 
Given a vector-valued Radon measure $\mu$ in $\mathbb{R}^{m}$, we denote by $T_{\mu}$ the $(m-1)$-dimensional current defined by the formula

$$
\left\langle T_{\mu}, \omega\right\rangle:=\int_{\operatorname{supp} \mu} w_{\omega} \cdot d \mu
$$

for each $C^{\infty}(m-1)$-form $\omega$ in $\mathbb{R}^{m}$ with compact support that is defined by (4.1). Obviously $T_{-\mu}=-T_{\mu}$. For a continuous $(m-1)$-form $\omega$ in $\mathbb{R}^{m}$ with compact support defined by (4.1), we let

$$
\int_{T_{\mu}} \omega:=\int_{\operatorname{supp} \mu} w_{\omega} \cdot d \mu
$$

in particular, $\int_{T_{\mu}} \omega=\left\langle T_{\mu}, \omega\right\rangle$ whenever $\omega$ is $C^{\infty}$.

Let $\omega$ be a $C^{\infty}(m-1)$-form in $\mathbb{R}^{m}$ with compact support defined by (4.1), and let $T:=\mathbf{E}^{m} \mathbf{L} g$ be in $\mathbf{N}_{m}^{c, \infty}\left(\mathbb{R}^{m}\right)$. Since $\partial T=-T_{D g}=T_{-D g}$ according to [5, Section 4.5.7], we obtain immediately

$$
\int_{T} d \omega=\langle T, d \omega\rangle=\langle\partial T, \omega\rangle=\int_{\partial T} \omega
$$

Our goal is to extend the Stokes equality $\int_{T} d \omega=\int_{\partial T} \omega$ to every continuous $(m-1)$-form $\omega$ that is pointwise Lipschitz outside a thin set.

Proposition 4.4. If $g \in B V_{c}^{\infty}\left(\mathbb{R}^{m}\right)$, then

$$
\int_{\operatorname{supp} D g} w \cdot d(D g)=-\int_{\mathbb{R}}\left(\int_{\partial_{*}\{g>t\}} w \cdot \nu_{\{g>t\}} d \mathcal{H}^{m-1}\right) d t
$$

for each continuous vector field $w: \operatorname{cl}\{g \neq 0\} \rightarrow \mathbb{R}^{m}$.

Proof. If $w: \mathbb{R}^{m} \rightarrow \mathbb{R}^{m}$ is $C^{\infty}$ and has compact support, then

$$
\int_{\mathbb{R}^{m}} \operatorname{div} w(x) d x=0
$$

by the Gauss-Green theorem. Fubini's theorem implies

$$
\int_{\mathbb{R}^{m}} g^{+}(x) \operatorname{div} w(x) d x=\int_{0}^{\infty}\left(\int_{\{g>t\}} \operatorname{div} w(x) d x\right) d t .
$$

Applying Fubini's theorem again and using (4.2), 


$$
\begin{aligned}
\int_{\mathbb{R}^{m}} g^{-}(x) \operatorname{div} w(x) d x & =\int_{0}^{\infty}\left(\int_{\{-g \geq t\}} \operatorname{div} w(x) d x\right) d t \\
& =\int_{0}^{\infty}\left(\int_{\mathbb{R}^{m}-\{g>-t\}} \operatorname{div} w(x) d x\right) d t \\
& =\int_{0}^{\infty}\left(\int_{\{g>-t\}} \operatorname{div} w(x) d x\right) d t \\
& =-\int_{-\infty}^{0}\left(\int_{\{g>t\}} \operatorname{div} w(x) d x\right) d t
\end{aligned}
$$

The previous two equalities together with (2.1) and (2.2) yield

$$
\begin{aligned}
\int_{\mathbb{R}^{m}} w \cdot d(D g) & =-\int_{\mathbb{R}^{m}} g(x) \operatorname{div} w(x) d x=-\int_{\mathbb{R}}\left(\int_{\{g>t\}} \operatorname{div} w(x) d x\right) \\
& =-\int_{\mathbb{R}}\left(\int_{\partial_{*}\{g>t\}} w \cdot \nu_{\{g>t\}} d \mathcal{H}^{m-1}\right) d t .
\end{aligned}
$$

Now suppose $w: \operatorname{cl}\{g \neq 0\} \rightarrow \mathbb{R}^{m}$ is continuous. Extend $w$ to a continuous vector field $v: \mathbb{R}^{m} \rightarrow \mathbb{R}^{m}$ with compact support, and find a sequence $\left\{v_{k}\right\}$ of $C^{\infty}$ vector fields with compact support defined on $\mathbb{R}^{m}$ that converges to $v$ uniformly. As $D g$ is a finite measure,

$$
\int_{\mathbb{R}^{m}} v \cdot d(D g)=\lim \int_{\mathbb{R}^{m}} v_{k} \cdot d(D g)
$$

by the dominated convergence theorem. Letting

$$
J(t):=\int_{\partial_{*}\{g>t\}} v \cdot \nu_{\{g>t\}} d \mathcal{H}^{m-1} \text { and } \quad J_{k}(t):=\int_{\partial_{*}\{g>t\}} v_{k} \cdot \nu_{\{g>t\}} d \mathcal{H}^{m-1},
$$

observe that

$$
\left|J(t)-J_{k}(t)\right|=\left|\int_{\partial_{*}\{g>t\}}\left(v-v_{k}\right) \cdot \nu_{\{g>0\}} d \mathcal{H}^{m-1}\right| \leq\|\{g>t\}\| \cdot\left|v-v_{k}\right|_{\infty}
$$

for $k=1,2, \ldots$, and $\mathcal{L}^{1}$-almost all $t \in \mathbb{R}$. In view of the coarea theorem for $\mathrm{BV}$ functions [4, Section 5.5], the dominated convergence theorem yields

$$
\int_{\mathbb{R}} J(t) d t=\lim \int_{\mathbb{R}} J_{k}(t) d t
$$


Combining $(*)$ and $(* *)$ with the first part of the proof,

$$
\int_{\mathbb{R}^{m}} v \cdot d(D g)=\int_{\mathbb{R}}\left(\int_{\partial_{*}\{g>t\}} v \cdot \nu_{\{g>t\}} d \mathcal{H}^{m-1}\right) d t .
$$

Since $\partial_{*}\{g>t\} \cup \operatorname{supp} D g \subset \operatorname{cl}\{g \neq 0\}$, the proposition follows.

Theorem 4.5. Let $E$ be a locally $B V$ set, and let $\omega$ be a continuous $(m-1)$ form in cl $E$. If $\omega$ is Lipschitz at each $x \in E-S$ where $S$ is a thin set, then $d \omega$ is R-integrable in $E$ and $\int_{T} d \omega=\int_{\partial T} \omega$ for each $T \in \mathbf{N}_{m}^{c, \infty}(E)$.

Proof. Let $w_{\omega}$ be the vector field associated with $\omega$ according to (4.1), and choose an $m$-dimensional current $T:=\mathbf{E}^{m} \mathbf{L} g$ in $\mathbf{N}_{m}^{c, \infty}(E)$. In view of linearity, we may assume that $g \geq 0$. According to Theorem 3.3, the divergence div $w_{\omega}$ of $w_{\omega}$ relative to $E$ is R-integrable in $E$, which means $d \omega$ is R-integrable in $E$. Denote by $F$ the R-primitive of $\operatorname{div} w_{\omega}$, and find a bounded BV set $A \subset E$ containing $\{g \neq 0\}$. Theorems 3.3 and 4.3 together with Proposition 4.4 imply

$$
\begin{aligned}
\int_{T} d \omega & =\int_{A} g(x) \operatorname{div} w_{\omega}(x) d x=(F \mathrm{~L} g)(A)=\langle F, g\rangle \\
& =\int_{0}^{\infty} F(\{g>t\}) d t=\int_{0}^{\infty}\left(\int_{\{g>t\}} \operatorname{div} w_{\omega} d \mathcal{L}^{m}\right) d t \\
& =\int_{0}^{\infty}\left(\int_{\partial_{*}\{g>t\}} v_{\omega} \cdot \nu_{\{g>t\}} d \mathcal{H}^{m-1}\right) d t \\
& =-\int_{\operatorname{supp} D g} w_{\omega} \cdot d(D g)=\int_{\operatorname{supp}(-D g)} w_{\omega} \cdot d(-D g)=\int_{\partial T} \omega .
\end{aligned}
$$

\section{Change of Variables}

A local lipeomorphism of a set $E \subset \mathbb{R}^{m}$ is a proper Lipschitz map $\phi: E \rightarrow \mathbb{R}^{m}$ having the following property: for each $x \in \mathrm{cl} E$ there is an $r>0$ such that $\phi$ restricted to $E \cap B(x, r)$ is a lipeomorphism. Note that $\phi$ has a unique Lipschitz extension $\bar{\phi}: \operatorname{cl} E \rightarrow \mathbb{R}^{m}$, and that proper means $\bar{\phi}^{-1}(K)$ is a compact set for each compact set $K \subset \mathbb{R}^{m}$. A simple compactness argument shows that for each compact set $C \subset \mathrm{cl} E$ there is a positive integer $n=n(C)$ such that the map $\bar{\phi}$ restricted to $C$ is at most $n$-to-one. In particular, $\bar{\phi}$ is a finite-to-one map.

Throughout the remainder of this paper, we select a fixed locally bounded BV set $E \subset \mathbb{R}^{m}$, and a local lipeomorphism $\phi: E \rightarrow \mathbb{R}^{m}$. If $g \in B V_{c}^{\infty}\left(\mathbb{R}^{m}\right)$, 
then

$$
\operatorname{deg}(\phi, g)(y):=\sum_{x \in \phi^{-1}(y)} g(x) \operatorname{sign} \operatorname{det} D \phi(x) .
$$

is defined for almost all $y \in \mathbb{R}^{m}$ by Rademacher's theorem [4, Section 3.1.2]. Given $\mathbf{E}^{m} \mathbf{L} g$ in $\mathbf{N}_{m}^{c, \infty}(E)$, it follows from [5, Section 4.1] that

$$
\phi_{\#}\left(\mathbf{E}^{m} \mathbf{L} g\right)=\mathbf{E}^{m} \operatorname{Ldeg}(\phi, g) .
$$

As $g$ has compact support, $\{\operatorname{deg}(\phi, g) \neq 0\}$ is a bounded subset of $\phi(E)$; in particular, $\operatorname{deg}(\phi, g)$ has compact support. Since there is an integer $n \geq 1$ such that $\phi$ is at most $n$-to-one on $\{g \neq 0\}$, the function $\operatorname{deg}(\phi, g)$ is essentially bounded. Finally, as $\mathbf{E}^{m} \mathbf{L} g$ is a normal $m$-dimensional current, so is $\mathbf{E}^{m} \operatorname{Ldeg}(\phi, g)$ by [5, Section 4.1]. Moreover, it follows from [5, Section 4.5] that $\mathbf{E}^{m} \mathbf{L} \operatorname{deg}(\phi, g)$ belongs to $\mathbf{N}_{m}^{c, \infty}[\phi(E)]$. The linear map

$$
\phi_{\#}: \mathbf{E}^{m} \mathbf{L} g \mapsto \mathbf{E}^{m} \operatorname{Ldeg}(\phi, g): \mathbf{N}_{m}^{c, \infty}(E) \rightarrow \mathbf{N}_{m}^{c, \infty}[\phi(E)]
$$

is $\tau$-continuous. Indeed, if $c$ is the Lipschitz constant of $\phi$ and $\mathbf{M}(T)$ denotes the mass of a current $T$ as defined in [5, Section 4.1.7], then

$$
\begin{aligned}
|\operatorname{deg}(\phi, g)|_{1} & =\mathbf{M}\left[\mathbf{E}^{m} \mathbf{L} \operatorname{deg}(\phi, g)\right]=\mathbf{M}\left[\phi_{\#}\left(\mathbf{E}^{m} \mathbf{L} g\right)\right] \\
& \leq c^{m} \mathbf{M}\left(\mathbf{E}^{m} \mathbf{L} g\right)=c^{m}|g|_{1}, \\
\|\operatorname{deg}(\phi, g)\| & =\mathbf{M}\left(\partial\left[\mathbf{E}^{m} \mathbf{L} \operatorname{deg}(\phi, g)\right]\right)=\mathbf{M}\left[\partial \phi_{\#}\left(\mathbf{E}^{m} \mathbf{L} g\right)\right] \\
& =\mathbf{M}\left(\phi_{\#}\left[\partial\left(\mathbf{E}^{m} \mathbf{L} g\right)\right]\right) \leq c^{m-1} \mathbf{M}\left[\partial\left(\mathbf{E}^{m} \mathbf{L} g\right)\right]=c^{m-1}\|g\| .
\end{aligned}
$$

If $A$ is a bounded BV set, we write $\mathbf{E}^{m} \mathbf{L} A$ and $\operatorname{deg}(\phi, A)$ instead of $\mathbf{E}^{m} \mathbf{L} \chi_{A}$ and $\operatorname{deg}\left(\phi, \chi_{A}\right)$, respectively.

Proposition 5.1. The set $\phi(E)$ is locally $B V$, and if $A \subset E$ is a bounded $B V$ set, then so is $\phi(A)$.

Proof. Choose a bounded BV set $A \subset E$, and an $x \in \operatorname{cl} A$. There is an $r>0$ so that $\phi$ is a lipeomorphism on the BV set $B=A \cap B(x, r)$. Now

$$
\operatorname{deg}(\phi, B)(y)= \begin{cases}\operatorname{sign} \operatorname{det} D \phi\left[\phi^{-1}(y)\right] & \text { if } y \in \phi(B), \\ 0 & \text { if } y \in \mathbb{R}^{m}-\phi(B),\end{cases}
$$

and $\operatorname{det} \phi(x) \neq 0$ for almost all $x \in B$. Thus $\chi_{\phi(B)}=|\operatorname{deg}(\phi, B)|$ almost everywhere. Since $\mathbf{E}^{m} \mathbf{L} \operatorname{deg}(\phi, B)=\phi_{\#}\left(\mathbf{E}^{m} \mathbf{L} B\right)$ belongs to $\mathbf{N}_{m}^{c, \infty}[\phi(E)]$, we 
infer that $\phi(B)$ is a bounded $\mathrm{BV}$ set. As $\operatorname{cl} A$ is compact, the set $\phi(A)$ is the union of a finite family of bounded BV sets. This implies $\phi(A)$ is itself a bounded BV set.

As $\phi$ is a proper map, given $r>0$, there is an $s>0$ such that $\phi^{-1}[\phi(E) \cap$ $B(0, r)]$ is contained in the bounded $\mathrm{BV}$ set $E \cap B(0, s)$. By the first part of the proof $D:=\phi[E \cap B(0, s)]$ is a bounded BV subset of $\phi(E)$ containing $\phi(E) \cap B(0, r)$. Hence

$$
\phi(E) \cap B(0, r)=D \cap \phi(E) \cap B(0, r)=D \cap B(0, r)
$$

is a $\mathrm{BV}$ set, and the proposition follows from the arbitrariness of $r$.

For a charge $F$ in $E$ and $\mathbf{E}^{m} \mathbf{L} g$ in $\mathbf{N}_{m}^{c, \infty}(E)$, let

$$
\left\langle F, \mathbf{E}^{m} \mathrm{~L} g\right\rangle=\langle F, g\rangle \text {. }
$$

It follows from Proposition 4.2 that $\mathbf{E}^{m} \mathbf{L} g \mapsto\left\langle F, \mathbf{E}^{m} \mathbf{L} g\right\rangle$ is a $\tau$-continuous linear functional on $\mathbf{N}_{m}^{c, \infty}(E)$, and that all $\tau$-continuous linear functionals on $\mathbf{N}_{m}^{c, \infty}(E)$ have this form. Consequently the dual map $\phi^{\#}$ of $\phi_{\#}$, defined by the identity

$$
\left\langle\phi^{\#} F, \mathbf{E}^{m} \mathbf{L} g\right\rangle=\left\langle F, \phi_{\#}\left(\mathbf{E}^{m} \mathbf{L} g\right)\right\rangle,
$$

maps charges in $\phi(E)$ to charges in $E$.

Proposition 5.2. If $F$ is a charge in $\phi(E)$, then $\phi^{\#} F(A)=\langle F, \operatorname{deg}(\phi, A)\rangle$ for each $A \in \mathcal{B V}\left(\mathbb{R}^{m}\right)$. If $E$ is a bounded $B V$ set and $\phi$ is a lipeomorphism, then $\phi^{\#} F(A)=[F \mathrm{~L} \operatorname{deg}(\phi, E)][\phi(A)]$ for each $A \in \mathcal{B V}(E)$.

Proof. Let $A$ be a bounded BV set, and $B=A \cap E$. Identities (5.1)-(5.3) yield

$$
\begin{aligned}
\phi^{\#} F(A) & =\phi^{\#} F(B)=\left\langle\phi^{\#} F, \chi_{B}\right\rangle=\left\langle\phi^{\#} F, \mathbf{E}^{m} \mathbf{L} B\right\rangle=\left\langle F, \phi_{\#}\left(\mathbf{E}^{m} \mathbf{L} B\right)\right\rangle \\
& =\left\langle F, \mathbf{E}^{m} \mathbf{L} \operatorname{deg}(\phi, B)\right\rangle=\langle F, \operatorname{deg}(\phi, B)\rangle=\langle F, \operatorname{deg}(\phi, A)\rangle .
\end{aligned}
$$

If $E$ is a bounded BV set and $\phi$ is a lipeomorphism, then

$$
\operatorname{deg}(\phi, A)= \begin{cases}\operatorname{sign} \operatorname{det} D \phi\left[\phi^{-1}(y)\right] & \text { if } y \in \phi(A \cap E) \\ 0 & \text { otherwise }\end{cases}
$$

for each bounded BV set $A$ and almost all $y \in \mathbb{R}^{m}$; in particular, this is true when $A=E$. Thus for every bounded BV set $A$, $\operatorname{deg}(\phi, A)=\operatorname{deg}(\phi, E) \chi_{\phi(A)}$. An application of the first part of the proof completes the argument. 
Proposition 5.3. If $F$ is an $A C_{*}$ charge in $\phi(E)$, then $\phi^{\#} F$ is an $A C_{*}$ charge in $E$ and $\left(\phi^{\#} F\right)^{\prime}(x)=F^{\prime}[\phi(x)] \operatorname{det} \phi(x)$ for almost all $x \in E$.

Proof. Cover cl $E$ by open balls $B_{1}, B_{2}, \ldots$ so that $\phi$ is a lipeomorphism on each $B_{i} \cap E$. For $i=1,2, \ldots$, let

$$
\phi_{i}:=\phi \uparrow\left(B_{i} \cap E\right) \quad \text { and } \quad F_{i}:=F \mathrm{~L} \phi_{i}\left(B_{i} \cap E\right) .
$$

By our assumption, the charge $F_{i}$ is $\mathrm{AC}_{*}$ in $\phi_{i}\left(B_{i} \cap E\right)$, and by (5.1) and Theorem 4.3, so is the charge $G_{i}:=F_{i} \mathrm{~L} \operatorname{deg}\left(\phi_{i}, B_{i} \cap E\right)$. Proposition 5.2 implies $\phi_{i}^{\#} F_{i}(A)=G_{i}[\phi(A)]$ for each BV set $A \subset B_{i} \cap E$. According to [1, Propositions 3.7 and 4.14], the charge $\phi_{i}^{\#} F_{i}$ is $\mathrm{AC}_{*}$ in $B_{i} \cap E$ and

$$
\left(\phi_{i}^{\#} F_{i}\right)^{\prime}(x)=G_{i}^{\prime}\left(\phi_{i}(x)\right)\left|D \phi_{i}(x)\right|
$$

for almost all $x \in B_{i} \cap E$.

As $\phi_{i}^{-1}(y)=B_{i} \cap \phi^{-1}(y)$ for each $y \in \mathbb{R}^{m}$, we have $\operatorname{deg}\left(\phi_{i}, A\right)=\operatorname{deg}(\phi, A \cap$ $B_{i}$ ) for each bounded BV set $A$. Thus if $G:=\phi^{\#} F$, then Proposition 5.2 implies $G \mathrm{~L} B_{i}=\phi^{\#} F_{i}$. Consequently, $G \mathbf{L} B_{i}$ is $\mathrm{AC}_{*}$ in $B_{i} \cap E$. Choose a negligible set $N \subset \mathrm{cl}_{*} E$. Since each $B_{i}$ is an open set, it is easy to see that

$$
V_{*} G\left(N \cap B_{i}\right)=V_{*}\left(G\left\llcorner B_{i}\right)\left(N \cap B_{i}\right)=0\right.
$$

for $i=1,2, \ldots$ As $N \subset \bigcup_{i} B_{i}$, we conclude that $G$ is $\mathrm{AC}_{*}$ in $E$.

Fix a ball $B_{i}$, and choose an $x \in B_{i} \cap E$ so that (5.4) holds, and the derivatives $G^{\prime}(x)$ and $F^{\prime}[\phi(x)]=F_{i}^{\prime}\left[\phi_{i}(x)\right]$ exist. In view of the first part of the proof and [3, Lemma 7.10], almost all $x \in B_{i} \cap E$ have these properties. As $B_{i}$ is an open set, $G^{\prime}(x)=\left(G \mathbf{L} B_{i}\right)^{\prime}(x)$. Now by (5.4) and Theorem 4.3,

$$
\begin{aligned}
G^{\prime}(x) & =\left(\phi_{i}^{\#} F_{i}\right)^{\prime}(x)=G_{i}^{\prime}\left(\phi_{i}(x)\right)\left|D \phi_{i}(x)\right| \\
& =F_{i}^{\prime}\left[\phi_{i}(x)\right] \operatorname{deg}\left(\phi_{i}, B_{i} \cap E\right)\left[\phi_{i}(x)\right] \cdot\left|D \phi_{i}(x)\right| \\
& =F_{i}^{\prime}\left[\phi_{i}(x)\right] \operatorname{sign} \operatorname{det} D \phi_{i}(x)\left|D \phi_{i}(x)\right|=F^{\prime}[\phi(x)] \operatorname{det} D \phi(x) .
\end{aligned}
$$

As $E=\bigcup_{i}\left(B_{i} \cap E\right)$, the theorem follows.

Theorem 5.4. Let $g \in B V_{\mathrm{loc}}^{\infty}\left(\mathbb{R}^{m}\right)$, and let $f$ be an $R$-integrable function in $\phi(E)$. The following statements are true.

(i) The function $(f \circ \phi)(\operatorname{det} D \phi) g$ is R-integrable in $E$.

(ii) For each bounded $B V$ set $A \subset E$, the function $f \operatorname{deg}\left(\phi, g \chi_{A}\right)$ is $R$ integrable in $\phi(A)$ and

$$
\int_{\phi(A)} f(y) \operatorname{deg}\left(\phi, g \chi_{A}\right)(y) d y=\int_{A} f[\phi(x)] \operatorname{det} D \phi(x) g(x) d x .
$$


Proof. Denote by $F$ the R-primitive of $f$. According to Theorem 4.3 and Proposition 5.3, the charge $\phi^{\#} F \mathrm{~L} g$ is the R-primitive of $(f \circ \phi)(\operatorname{det} D \phi) g$. Choose a bounded BV set $A \subset E$ and observe that $G:=F \operatorname{Ldeg}\left(\phi, g \chi_{A}\right)$ is the R-primitive of $f \operatorname{deg}\left(\phi, g \chi_{A}\right)$ by Theorem 4.3. Identities (5.1)-(5.3) imply

$$
\begin{aligned}
{\left[\left(\phi^{\#} F\right) \mathrm{L} g\right](A) } & =\left\langle\phi^{\#} F, g \chi_{A}\right\rangle=\left\langle\phi^{\#} F, \mathbf{E}^{m} \mathrm{~L}\left(g \chi_{A}\right)\right\rangle \\
& =\left\langle F, \phi_{\#}\left[\mathbf{E}^{m} \mathrm{~L}\left(g \chi_{A}\right)\right]\right\rangle=\left\langle F, \mathbf{E}^{m} \mathrm{~L} \operatorname{deg}\left(\phi, g \chi_{A}\right)\right\rangle \\
& =\left\langle F, \operatorname{deg}\left(\phi, g \chi_{A}\right)\right\rangle=\left\langle F, \operatorname{deg}\left(\phi, g \chi_{A}\right) \chi_{\phi(A)}\right\rangle=G[\phi(A)],
\end{aligned}
$$

and the theorem follows.

In analogy with $C^{\infty} m$-forms, if $\theta=f d x$ is any $m$-form in $\phi(E)$, we let

$$
\phi^{\#} \theta:=(f \circ \phi) \operatorname{det} D \phi d x .
$$

The $m$-form $\phi^{\#} \theta$ is defined almost everywhere in $E$.

Corollary 5.5. If $\theta$ is an R-integrable $m$-form in $\phi(E)$, then $\phi^{\#} \theta$ is an $R$ integrable $m$-form in $E$, and for each $m$-dimensional current $T \in \mathbf{N}_{m}^{c, \infty}(E)$ we have $\int_{T} \phi^{\#} \theta=\int_{\phi_{\#} T} \theta$.

Proof. Let $\theta:=f d x$, and choose a $T:=\mathbf{E}^{m} \operatorname{L} g$ in $\mathbf{N}_{m}^{c, \infty}(E)$. Then

$$
\phi^{\#} \theta=(f \circ \phi) \operatorname{det} D \phi d x \quad \text { and } \quad \phi_{\#} T=\mathbf{E}^{m} \mathrm{~L} \operatorname{deg}(\phi, g) .
$$

If $A \subset E$ is a bounded BV set containing $\{g \neq 0\}$, then $\phi(A)$ is a bounded BV subset of $\phi(A)$ containing $\{\operatorname{deg}(\phi, g) \neq 0\}$. As $g \chi_{A}=g$, Theorem 5.4 implies

$$
\begin{aligned}
\int_{T} \phi^{\#} \theta & =\int_{A} f[\phi(x)] \operatorname{det} D \phi(x) g(x) d x \\
& =\int_{\phi(A)} f(y) \operatorname{deg}(\phi, g)(y) d y=\int_{\phi_{\#} T} \theta .
\end{aligned}
$$

Remark 5.6. A proper Lipschitz map $\psi: A \rightarrow \mathbb{R}^{m}$ of a set $A \subset \mathbb{R}^{m}$ is called

- regular if it is injective, $\psi^{-1}(S)$ is a thin set whenever $S \subset \mathbb{R}^{m}$ is a thin set, and $|\psi(B)| \geq c|B|$ for a $c>0$ and all $B \subset A$.

- locally regular if for each $x \in \operatorname{cl} A$ there is an $r>0$ such that $\psi$ restricted to $A \cap B(x, r)$ is regular.

Clearly, each local lipeomorphism is a regular map, but the converse is false. Notwithstanding, it is easy to show that the results of Section 5 , together with their proofs, remain valid when the fixed local lipeomorphism $\phi: E \rightarrow \mathbb{R}^{m}$ is replaced by a locally regular map. 


\section{References}

[1] Z. Buczolich and W. F. Pfeffer, Variations of additive functions, Czechoslovak Math. J., 47 (1997), 525-555.

[2] Z. Buczolich and W. F. Pfeffer, On absolute continuity, J. Math. Anal. Appl., 222 (1998), 64-78.

[3] Z. Buczolich, T. De Pauw and W. F. Pfeffer Charges, BV functions, and multipliers for generalized Riemann integrals, Indiana Univ. Math. J., 48 1999, 1471-1511.

[4] L. C. Evans and R. F. Gariepy, Measure Theory and Fine Properties of Functions, CRC Press, Boca Raton, 1992.

[5] H. Federer, Geometric Measure Theory, Springer-Verlag, New York, 1969.

[6] W. F. Pfeffer, The Gauss-Green theorem, Adv. Math., 87 (1991), 93-147.

[7] W. F. Pfeffer, The Riemann Approach to Integration, Cambridge Univ. Press, Cambridge, 1993.

[8] M. Spivak, Calculus on Manifolds, Benjamin, London, 1965.

[9] W. P. Ziemer, Weakly Differentiable functions, Springer-Verlag, New York, 1989. 